\title{
Effects of Obstacles on Moving Particles in Rotary Kiln*
}

\author{
By Shin TSUKANO,** Akira MORIYAMA, ${ }^{* *}$ Kazuo ARAKI** \\ and Shigekatsu MORI**
}

\begin{abstract}
Synopsis
Effects of obstacles put in the moving particle bed in rotary kiln can be divided into the one on the path and the other on the speed of displacement of the individual particle. The former effect results in distortion or shift of the circulating path. To estimate the shift of the cascading path of particles the concept of "transition probability" was taken, which was found to be well expressed by gamma distribution law with and without obstacles.

The latter effect on the speed was always retardation. Adequate obstacles are to be regarded as effective means for controlling the residence time of particles in rotary kiln.
\end{abstract}

\section{Introduction}

Not any investigation of effect of obstacles, independently sustained off the rotating wall, on the moving particle bed of rotary kiln has been known. The situation, for example, occurs in temperature measurements of the particle bed by means of thermocouples in laboratory and industrial kiln. ${ }^{1)}$ A thorough measurement of the temperature distribution in the moving particle bed for study on heat transfer in the kiln showed that there was an appreciable temperature gradient across the particle bed and also a considerable disturbance due to the insertion of thermocouples in the bed. Present work is concerned with the disturbing effect of the obstacle independently sustained off the rotating wall, with which the disturbing effect of inserted elements such as thermocouples in rotary kiln is generalized.

Behavior of the moving particles in rotary kiln can be treated conveniently dividing into the path and the speed. At a slower rotation, the circulating path of the moving particles is separated into the rotation path being subject to the wall rotation and the cascading path at the superficial layer of the particle bed. According to the experiment by Suga and Moriyama ${ }^{2)}$ with a drum rotating at 3 to 7 r.p.m. the transition probability between two fixed points of the rotation path ranges from 0.9 to 1.0 for every path, that is, there is few interchange of particles among each path, while the transition probability in the cascading path is widely distributed. And both the probabilities may be characteristic, corresponding to the operational conditions of the kiln.

Presently, the transition probability in the cascading is taken as a measure to estimate the disturbing effect of obstacles on the path of the moving particles. As far as the circulating speed of the particles is concerned, the rotation time and the cascading time, later defined, of the particle are discussed.

\section{Experimental Procedures}

A horizontal drum, $15.2 \mathrm{~cm}$ diameter and $25 \mathrm{~cm}$ long, made of transparent acrylic resine was availed as the model kiln. Particles were glass beads of $3 \mathrm{~mm}$ diameter. Measurement of the transition probability in the cascading path was done with the colored beads as the tracer, which were followed through a scaled plate. This is essentially identical to that by Suga and Moriyama, ${ }^{2)}$ except a halogen lamp equipped underneath the drum for insuring visibility within two or three surface layer of the particle bed. The upper and the lower half of the cascading plane were respectively divided into ten equal sections numbered from 1 to 10 outwards in sequence. When the respective interval numbers of the upper and the lower half of the cascading plane are designated as $i$ and $j$, the transition probability, $P_{i j}$, in the cascading path is defined as the fraction of the particles commencing the travel at the interval $i$ and leaving the path at the interval $j$ to those turning up on the plane at the interval $i$;

$$
\begin{aligned}
& \text { (Particles commencing the travel at } \\
& \text { the interval } i \text { and leaving the cas- } \\
& \text { cading plane at the interval } j \text { ) } \\
& \text { (Particles turning up on the cas- } \\
& \text { cading plane at the interval } i \text { ) }
\end{aligned}
$$

As experimentally confirmed, any particle turning up on the upper plane leaves the cascading path at some interval of the lower plane. Therefore, Eq. (2) can be written:

$$
\sum_{j=1}^{10} P_{i j}=1 \quad(i=1, \ldots, 10)
$$

With regard to the speed of the moving particles, the rotation and the cascading times are defined as those necessary for complete travelling of the tracer particle on the rotation path, arc $\mathrm{AB}$, and on the cascading path, chord BA, respectively specified in Fig. 1. Both times were measured by a stopwatch.

Three kinds of obstacles and four modes of their insertion were adopted in taking account of the arrangement of thermocouples for temperature measurements as shown in Fig. 2. With mode A only the wire level was varied, remaining the sustension rods fixed, as against entire insertion of obstacles with modes B, C and D.

Tracer experiments were all performed under a fixed condition of 4 r.p.m. and $5 \mathrm{~cm}$ deep particle bed. 


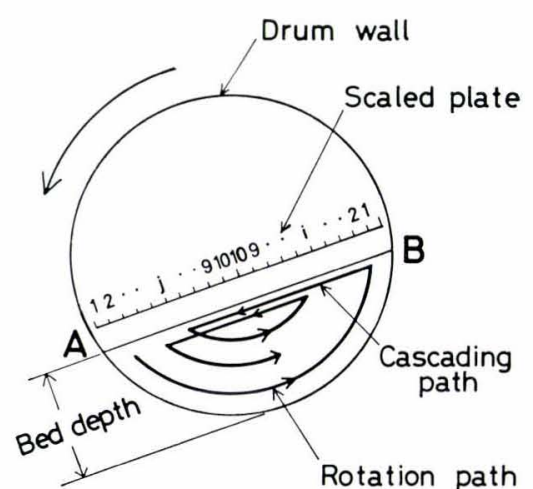

Fig. 1. Circulating paths of particles and interval numbers of cascading plane.
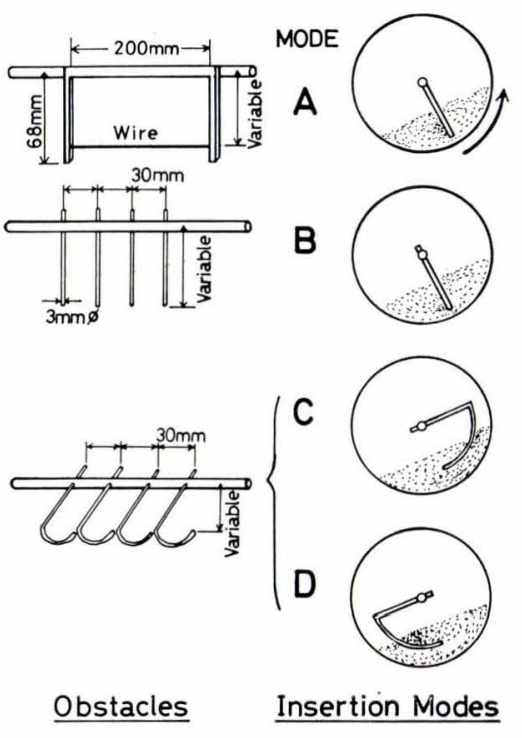

Fig. 2.

Obstacles and their insertion modes.

\section{Results and Discussion}

\section{Transition Probability in Cascading Path}

Typical results of the transition probability, $P_{i j}$, are shown in Fig. 3. Without obstacle the peak of the distribution curve of $P_{i j}$ tends to shift toward the larger $j$ with the larger $i$ and this is also the case by Suga and Moriyama. ${ }^{2)}$ In contrast to the case that the obstacle is put in the particle bed, the peak of the distribution curve of $P_{i j}$ is always near the interval $j=1$; particles coming into the cascading path almost slide down to the lowest end of the path.* Results in the figure show that the distribution curve with the obstacle of the shallower insertion approaches closer to that without obstacle.

In order to appreciate the disturbing effect of obstacles based on the experimental results, formulation of $P_{i j}$ is necessary. After examinations of the descriptabilities of several distribution laws, gamma distribution law was the best with and without the In Fig. 4 the observed and the calculated distribuobstacle, regardless of its mode and insertion depth.

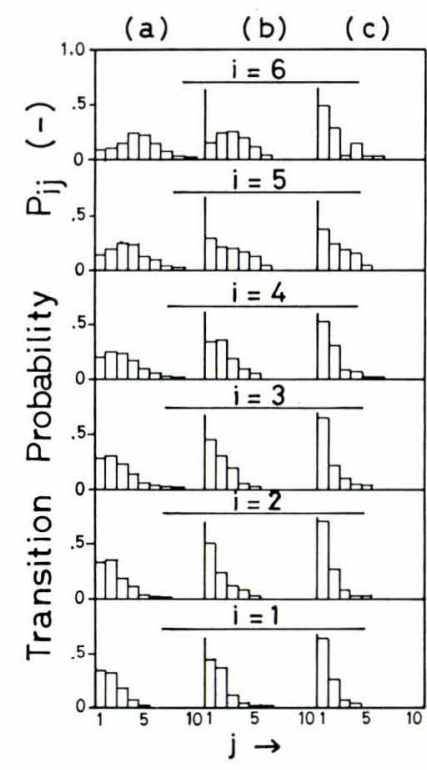

(a) without obstacle

(b) with obstacle mode B (insertion depth $1 \mathrm{~cm}$ )

(c) with obstacle mode B (insertion depth $4 \mathrm{~cm}$ )

Fig. 3. Transition probabilities in cascading path.

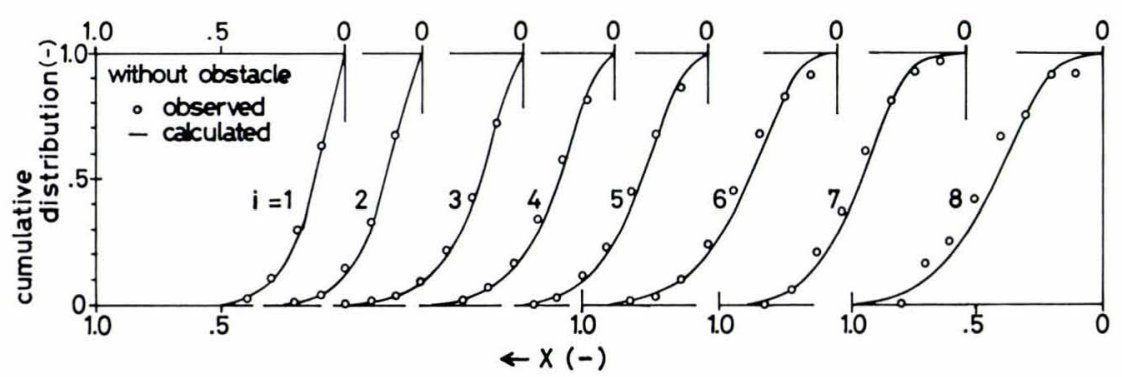

Fig. 4. Observed and calculated distributions in cascading path.

tions, as an example, are compared and the agreement is satisfactory. It was confirmed that all observations along with the results by Suga and Moriyama ${ }^{2)}$ were well described with gamma distribution law as expressed in Eq. (3).

$$
\begin{aligned}
& f(x)=\left\{\begin{array}{cl}
\left\{\lambda^{m} / \Gamma(m)\right\} \cdot x^{m-1} \cdot \exp (-\lambda x), & x \geqq 0 \\
0 & , x<0
\end{array}\right. \\
& \Gamma(m)=\int_{0}^{\infty} u^{m-1} e^{-u} d u
\end{aligned}
$$

$m$ and $\lambda$ are necessary to calculate the theoretical distribution density function $f(x)$ in Eq. (3) and they can be given after substituting both mean $E$ and variance $\sigma^{2}$ of the observed $P_{i j}$ into Eqs. (5) and (6).

$$
\begin{aligned}
E & =m / \lambda . \\
\sigma^{2} & =m / \lambda^{2} .
\end{aligned}
$$

As is well known, $f(x) \Delta x$ is the fraction of the particles commencing the travel at the interval $i$ and leaving the path at the interval $x \sim x+\Delta x$ to those turning up

\footnotetext{
* This tendency is the most remarkable with insertion mode $\mathrm{A}$, while with modes $\mathrm{B}, \mathrm{C}$ and $\mathrm{D}$ rather similarly relaxed disturbances were observed.
} 
on the cascading plane at the interval $i$. That is,

$$
P_{i j}=\int_{x}^{x+\Delta x} f(x) d x
$$

The effect of the obstacle on the cascading path of the moving particles is to be reflected upon the parameters, $m$ and $\lambda$, all data of which are summarized in Table 1. Although further works should be required, obstacle insertions tend to make $m$ smaller, and any definite effect on $\lambda$ is not necessarily observed as far as in Table 1 .

\section{Rotation and Cascading Times}

Measurements of the rotation time, $T_{r}^{*}$, and the cascading time, $T_{c}^{*}$, are illustrated in Figs. 5 and 6 in which the abscissa is the depth of insertion of obstacles, $d_{I}$. Generally, as $d_{I}$ becomes larger, both the rotation and the cascading times increase remarkably; the speed of the moving particle is decreased. With decreasing $d_{I}$, both times approach to those without obstacle.

In rotary kilns without obstacles, particles are usually mixed only in the cascading plane. As mentioned above putting any obstacle in the particle bed causes disturbances not only in the cascading but in the rotation path of the particles. For example, the disturbed motions of the particles due to the insertion of a thermocouple is illustrated in Photo. 1. Since

Table 1. Parameters $m$ and $\lambda$ as measures of disturbing effect of obstacles.

\begin{tabular}{|c|c|c|c|c|c|c|c|c|c|c|c|c|}
\hline \multirow{2}{*}{ Mode } & \multirow{2}{*}{$d_{I}(\mathrm{~cm})$} & \multirow{2}{*}{ Parameter } & \multicolumn{10}{|c|}{$i$} \\
\hline & & & 1 & 2 & 3 & 4 & 5 & 6 & 7 & 8 & 9 & 10 \\
\hline \multirow{2}{*}{$\begin{array}{l}\text { Without } \\
\text { obstacle }\end{array}$} & \multirow{2}{*}{-} & $m$ & 2.11 & 2.12 & 2.15 & 2.65 & 3.25 & 4.44 & 7.23 & 5.15 & - & - \\
\hline & & $\lambda$ & 13.7 & 12.5 & 10.8 & 10.8 & 11.2 & 12.0 & 16.1 & 11.2 & - & - \\
\hline \multirow{8}{*}{ A } & \multirow{2}{*}{1} & $m$ & 1.17 & 1.56 & 1.56 & 1.33 & 2.19 & 2.17 & 2.24 & 2.50 & - & - \\
\hline & & $\lambda$ & 8.75 & 12.2 & 9.80 & 9.07 & 10.3 & 12.6 & 17.5 & 10.0 & - & - \\
\hline & \multirow{2}{*}{2} & $m$ & 1.91 & 1.62 & 2.20 & 2.06 & 2.16 & 3.01 & - & - & - & - \\
\hline & & $\lambda$ & 18.7 & 13.2 & 17.6 & 15.2 & 12.8 & 16.7 & - & - & - & - \\
\hline & \multirow{2}{*}{3} & $m$ & 2.23 & 1.58 & 1.31 & 1.41 & 1.36 & 1.69 & 1.95 & 3.77 & - & - \\
\hline & & $\lambda$ & 27.8 & 12.6 & 12.6 & 14.0 & 9.27 & 11.3 & 14.1 & 28.2 & - & - \\
\hline & \multirow{2}{*}{4} & $m$ & 2.11 & 1.88 & 3.17 & 2.14 & 2.29 & 1.18 & 2.04 & - & - & - \\
\hline & & $\lambda$ & 29.3 & 27.3 & 47.4 & 26.8 & 35.3 & 18.2 & 31.4 & - & - & - \\
\hline \multirow{8}{*}{ B } & \multirow{2}{*}{1} & $m$ & 1.83 & 1.50 & 2.09 & 2.18 & 2.26 & 3.38 & - & - & - & - \\
\hline & & $\lambda$ & 13.8 & 10.9 & 15.6 & 13.0 & 10.2 & 13.5 & - & - & - & - \\
\hline & \multirow{2}{*}{2} & $m$ & 1.48 & 1.87 & 2.31 & 2.20 & 2.48 & 2.53 & 2.99 & - & - & - \\
\hline & & $\lambda$ & 12.1 & 13.1 & 16.5 & 13.9 & 14.1 & 13.0 & 15.5 & - & - & - \\
\hline & \multirow{2}{*}{3} & $m$ & 1.97 & 2.05 & 1.82 & 1.60 & 1.66 & 1.83 & 3.13 & 9.38 & - & - \\
\hline & & $\lambda$ & 15.8 & 16.5 & 13.2 & 11.4 & 13.6 & 13.0 & 15.4 & 37.5 & - & - \\
\hline & \multirow{2}{*}{4} & $m$ & 1.65 & 1.35 & 1.25 & 1.52 & 1.80 & 1.25 & 2.58 & - & - & - \\
\hline & & $\lambda$ & 15.9 & 14.1 & 11.5 & 11.9 & 9.78 & 8.07 & 13.5 & - & - & - \\
\hline \multirow{8}{*}{$\mathrm{C}$} & \multirow{2}{*}{1} & $m$ & 1.60 & 1.39 & 1.96 & 1.75 & 2.28 & 2.46 & 4.27 & - & - & - \\
\hline & & $\lambda$ & 11.5 & 10.3 & 13.5 & 11.4 & 12.2 & 13.4 & 15.4 & - & - & - \\
\hline & \multirow{2}{*}{2} & $m$ & 1.52 & 1.80 & 1.53 & 1.90 & 2.77 & 2.84 & 4.47 & - & - & - \\
\hline & & $\lambda$ & 12.0 & 14.0 & 11.6 & 12.3 & 15.0 & 13.3 & 18.6 & - & - & - \\
\hline & \multirow{2}{*}{3} & $m$ & 1.72 & 1.81 & 1.60 & 1.80 & 1.83 & 2.04 & 2.53 & - & - & - \\
\hline & & $\lambda$ & 13.1 & 12.2 & 11.3 & 11.0 & 10.7 & 11.8 & 8.65 & - & - & - \\
\hline & \multirow{2}{*}{4} & $m$ & 1.59 & 1.50 & 1.51 & 1.95 & 1.69 & 1.81 & 1.60 & 1.34 & - & - \\
\hline & & $\lambda$ & 16.7 & 14.7 & 13.7 & 15.6 & 13.1 & 14.2 & 10.4 & 7.87 & - & - \\
\hline \multirow{8}{*}{$\mathrm{D}$} & \multirow{2}{*}{1} & $m$ & 1.76 & 1.84 & 2.33 & 1.67 & 2.46 & 2.57 & 3.20 & - & - & - \\
\hline & & $\lambda$ & 11.4 & 13.2 & 14.7 & 10.2 & 11.8 & 11.3 & 16.0 & - & - & - \\
\hline & \multirow{2}{*}{2} & $m$ & 1.92 & 1.93 & 2.19 & 1.97 & 1.95 & 2.18 & 6.51 & - & - & - \\
\hline & & $\lambda$ & 13.8 & 13.3 & 17.1 & 10.6 & 9.70 & 9.53 & 25.0 & - & - & - \\
\hline & \multirow{2}{*}{3} & $m$ & 2.00 & 1.89 & 1.82 & 1.55 & 1.81 & 1.29 & 1.91 & - & - & - \\
\hline & & $\lambda$ & 20.6 & 17.7 & 16.4 & 13.5 & 10.4 & 8.57 & 12.8 & - & - & - \\
\hline & 4 & $m$ & 1.22 & 1.60 & 0.939 & 1.11 & 1.07 & 1.33 & 1.20 & - & - & - \\
\hline & 4 & $\lambda$ & 16.3 & 23.8 & 11.6 & 13.8 & 11.6 & 11.6 & 7.76 & - & - & - \\
\hline
\end{tabular}




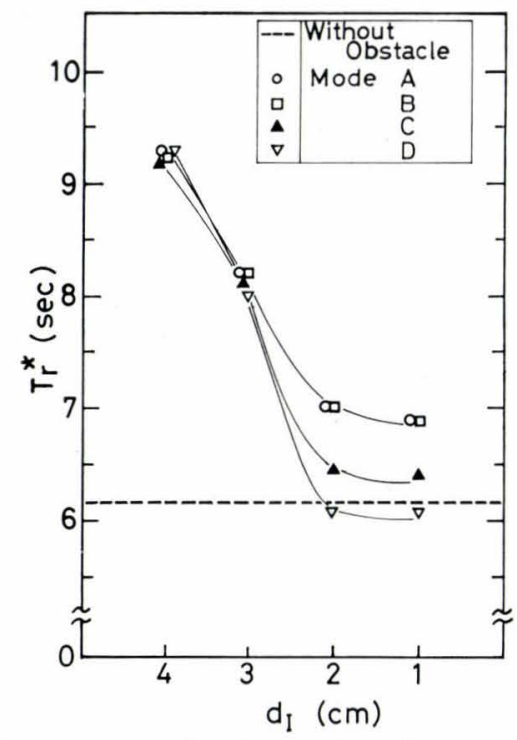

Fig. 5. Rotation time $v s$. insertion depth.

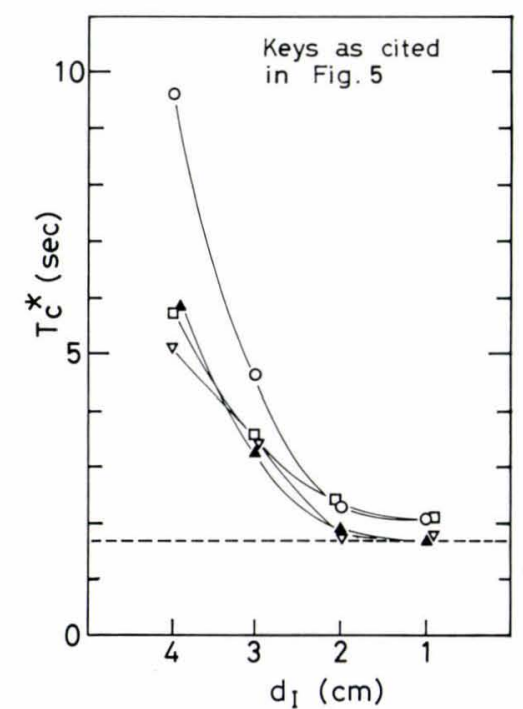

Fig. 6. Cascading time vs, insertion depth.

the disturbances of particle motions raised by obstacles, in turn, produce the disturbance of the temperature distribution in the particle bed, such as shown in Fig. 7, an attention must be paid to designing of the measuring system of temperature in experiments and in operations.

Effect of the obstacle is not always confined to the negative aspect cited above. As the disturbance of the particle motion usually brings about a reduction of the speed of particles, particles in a shorter kiln with the obstacle may have a similar extent of the residence time as in a larger kiln without obstacle. This is perhaps a positive aspect of the insertion of the obstacle; types, insertion modes and disturbance mechanisms of obstacles are to be studied.

\section{Conclusions}

The effect of obstacles put in the particle bed on the path and the speed of the particle motion in rotary kiln was investigated. Transition probability of particles in the cascading path is affected by the insertion

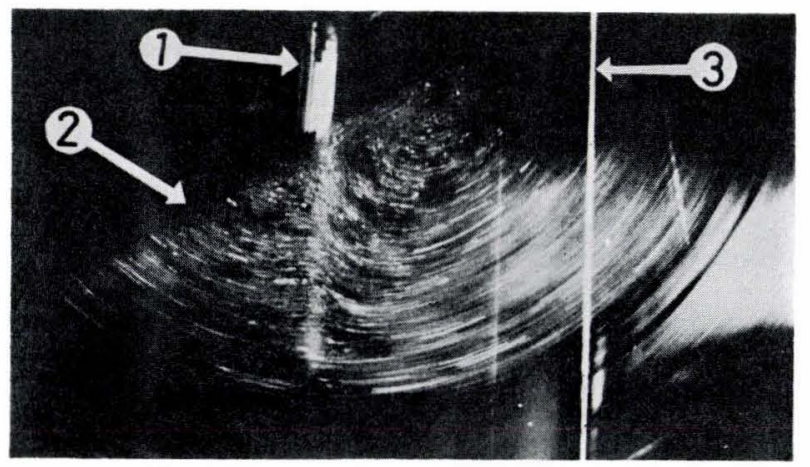

(1) thermocouple

(2) cascading plane

(3) vertical line

Photo. 1. Disturbed motion of particles due to insertion of thermocouple.

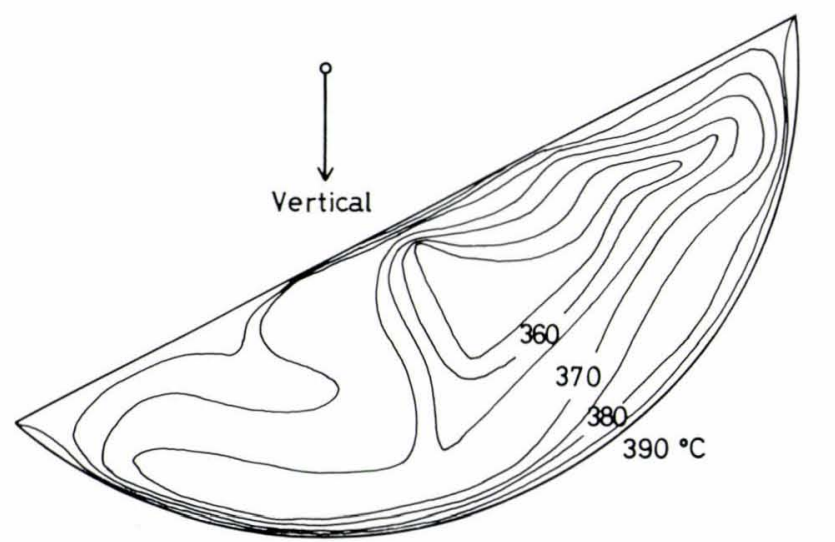

Fig. 7. An example of distorted temperature distribution in the particle bed measured with inserted thermocouple of mode B $(16.5 \mathrm{~cm}$ dia. steel drum, $0.1 \mathrm{~cm}$ dia. steel ball, $5 \mathrm{~cm}$ deep bed, outer wall temp. $400^{\circ} \mathrm{C}$ )

of obstacles, which tends to maximize the fractional particles entering into the rotation path at the lowest end of the cascading plane. It was found that the transition probability of the cascading could be well described by gamma distribution law with and without the obstacle, and parameters, $m$ and $\lambda$, of the distribution function might be availed for appreciations of the disturbing effect of the obstacle. With a small obstacle the speed of particle motion in the rotation and the cascading paths is significantly decreased.

The observed effects of the obstacle seem to suggest not only the negative aspect such as the disturbances of the path and the speed of the moving particles, but also a positive one such as an ability to increase the residence time of the particle in rotary kilns.

\section{Nomenclature}

$d_{I}$ : depth of obstacle put in particle layer $(\mathrm{cm})$

E: mean value, Eq. (3) (-)

$m$ : parameter of gamma distribution function, Eq. (1) (-)

$P_{i j}$ : transition probability of cascading (-)

$T_{c}^{*}: \quad$ cascading time $(\mathrm{sec})$

$T_{r}^{*}$ : rotation time $(\mathrm{sec})$ 
$x$ : dimensionless length $(-)$

$\Gamma(m)$ : gamma function, Eq. (2) (-)

$\lambda$ : parameter of gamma distribution function, Eq. (1) (-)

$\sigma^{2}: \quad$ variance, Eq. (4) (-)

\section{Acknowledgements}

This work was supported by Grant-in-Aid for
Scientific Research from Ministry of Education, No. 365240 .

\section{REFERENCES}

1) H. Moritomi, S. Mori, M. Sakaguchi, K. Araki and A. Moriyama: Tetsu-to-Hagané, 64 (1978), 1491.

2) T. Suga and A. Moriyama: Tetsu-to-Hagané, 60 (1974), 1155. 